\title{
Reach-to-grasp motions: Towards a dynamic classification approach for upper-limp prosthesis
}

\author{
I. Batzianoulis ${ }^{1}$, A. M. Simon ${ }^{2}$, L. Hargrove ${ }^{2}$, A. Billard ${ }^{1}$
}

\begin{abstract}
During reach-to-grasp motions, the Electromyographic (EMG) activity of the arm varies depending on motion stage. The variability of the EMG signals results in low classification accuracy during the reaching phase, delaying the activation of the prosthesis. To increase the efficiency of the pattern-recognition system, we investigate the muscle activity of four individuals with below-elbow amputation performing reach-to-grasp motions and segment the arm-motion into three phases with respect to the extension of the arm. Furthermore, we model the dynamic muscle contractions of each class with Gaussian distributions over the different phases and the overall motion. We quantify of the overlap among the classes with the Hellinger distance and notice larger values and, thus, smaller overlaps among the classes with the segmentation to motion phases. A Linear Discriminant Analysis classifier with phase segmentation affects positively the classification accuracy by $6-10 \%$ on average.

\section{INTRODUCTION}

Neuro-prosthetic devices can restore motor abilities lost after amputation and improve the quality of life for amputees. In the case of myo-prosthesis for the upper-limb, the device decodes the grasping intention from electromyography (EMG), and reacts accordingly. The seamless operation of the device requires an accurate identification of the user's intention from the pattern-recognition system. However, the stochastic nature of the EMG signals increases the challenge of the pattern-recognition system and constraints its performance. Low accuracy introduces delays in the operation of the myo-prosthesis and limits the coordination with the user's intention [1]. Thus, it is crucial to address the variability of EMG signals in order to increase the efficiency of the system.

Different arm positions and levels of activation are confounding factors that introduce signal variations and affect the performance of the pattern-recognition system [2], [3]. In addtition, the changes in EMG pattern characteristics during dynamic motions lowers the accuracy of the system [4]. The introduction of dynamic, and more complicated, training protocols improves the accuracy of the system by including larger contraction intervals [5] and containing the EMG activity of the complete motion. However, in long intervals the EMG signals become non-stationary and this is reflected in the variation on EMG patterns.

\footnotetext{
*This work has received funding from the Swiss National Science Foundation through the National Centre of Competence in Research (NCCR) in Robotics, the Hasler foundation and the United States National Institute of Health

${ }^{1}$ I. Batzianoulis and A. Billard are with Learning Algorithms and Systems Laboratory (LASA) at Ecole Polytechnique Federale de Lausanne (EPFL), Switzerland

${ }^{2}$ A. M. Simon and L. Hargrove are with the Center of Bionics Medicine at Shirley Ryan Abilitylab and the department of Mechanical Engineering of the Northwestern University, Chicago, Il, USA
}

To address the variability of the EMG signals over arm motions, the patterns over different motion phases should be further investigated, especially in individuals with amputation. The authors in [3] show that the muscle activation differs with respect to the arm position and that examining the EMG patterns is important. In our work, we elaborate on the EMG pattern during reach-to-grasp motions in individuals with amputation.

In this paper, we offer an approach to model the stochastic nature of the EMG pattern and relate this to the evolution of the muscular activity during the three typical phases underlying a reach and grasp motion. Specifically, we separate the motion into three phases and model the muscular activity of each class (i.e. grasp type) with Gaussians after performing Linear Discriminant Analysis (LDA). We analyse the result of the LDA projection and relate this to the muscular activity. Moreover, we examine the classification accuracy when training three LDA classifiers; one for each phase, and compare it with the accuracy of an LDA classifier over all phases. We evaluate the approach off-line with four individuals with transradial amputation.

\section{A. Experimental protocol}

$$
\text { II. METHODS }
$$

Four individuals with below the elbow amputation participated in the experiment. All the participants gave written consent, and experiments were performed at the Shirley Ryan Abilitylab in Chicago under a protocol approved by the Northwestern University Institutional Review Board (IRB) Two of the amputee subjects had undergone a TMR surgery, as in [1].

During the experiment, the subjects sat in front of a table, facing a computer screen, with their elbow at a $90^{\circ}$ angle. The subjects would start their self-paced motion when cued by the experimenter, grasping the object with their intact hand, and simultaneously imitating the motion with their phantom limb, see Figure 11. During the training phase, we recorded the EMG activity as well as the extension of the elbow joint. In a real-time control scenario, the same protocol would be followed for training the pattern recognition system.

\section{B. Apparatus}

Custom computer software [6] was used for signal acquisition, with EMG signals acquired at $1000 \mathrm{~Hz}$ with a $30-350 \mathrm{~Hz}$ band-pass filter using TI ADS1298 biosignal amplifiers. The EMG activity of 5 muscles of the residual arm was recorded: Flexor Digitorum Superficialis (FDS), Extensor Digitorum Communis (EDC), Flexor Carpi Ulnaris (FCU), Extensor Carpi Ulnaris (ECU), Flexor Carpi Radialis $(F C R)$. To construct a linear envelope, full-wave 

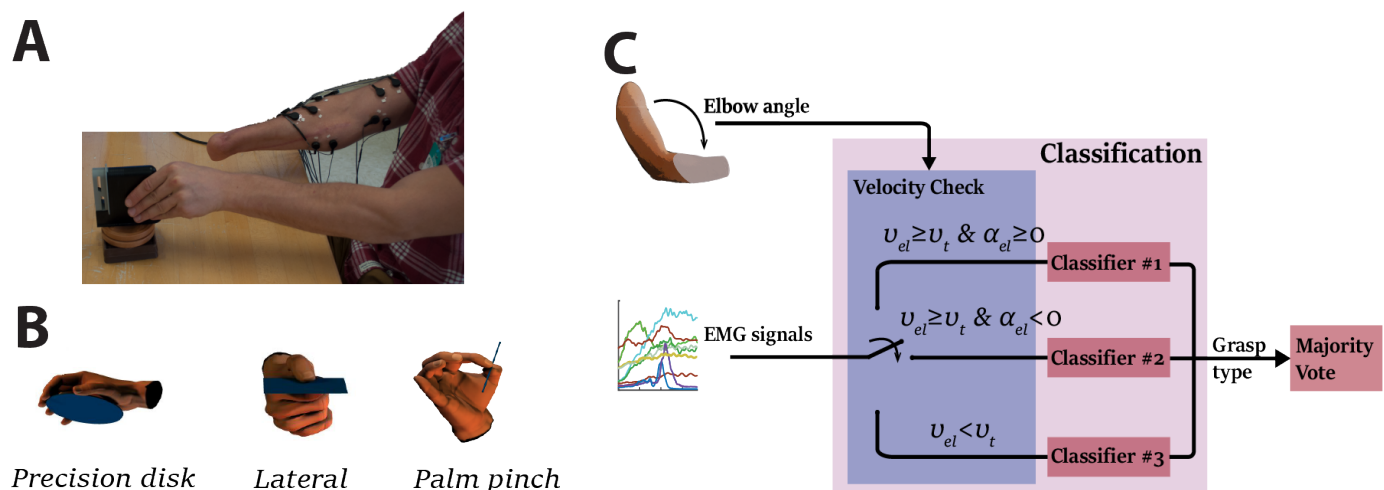

Fig. 1: a) Experimental set-up for training the system with amputee subjects in data recordings. EMG-information from the amputated arm are recorded while the subject performs the reach and grasp motion with his/her intact arm, b) The selected three grasp types used in our classification, following the names and using figures from the taxonomy of [7]. The variance indicates different muscle activation among the subjects, c) An illustration of the classification approach with one classifier per phase. The classifier is selected with respect of the angular velocity $\left(u_{e l}\right)$ and angular acceleration $\left(a_{e l}\right)$ of the elbow joint. For each time window, the angular velocity on the elbow $\left(u_{e l}\right)$ is compared with a velocity threshold $\left(u_{t}\right)$. If the angular velocity is less than the threshold, the arm has completed its extension and the classifier of the third phase is selected. Otherwise, the motion phase is defined by the angular acceleration $\left(a_{e l}\right)$. If the angular acceleration is greater than zero then the classifier of the first phase is selected. Accordingly, if the angular acceleration is less than zero then the classifier of the second phase is selected.

rectification was performed, followed by smoothing with a low-pass seventh-order Butterworth filter with cut-off frequency at $20 \mathrm{~Hz}$. Finally, each channel was normalized by the maximum value recorded across the trials. A goniometer was placed on the elbow for measuring the onset and extension of the elbow.

\section{Phases of the motion and classification method}

Taking inspiration from this behavior [8], we divided the reach-to-grasp motion into three phases with respect to the extension of the elbow joint. The first phase is defined as the interval from motion onset, i.e. when the angular velocity of the elbow joint exceeds a velocity threshold, until the angular velocity of the elbow reaches its maximum. The second phase is the interval between the aforementioned maximum angular velocity and the end of the reaching motion, i.e. when the angular velocity of the elbow drops below a velocity threshold. We defined the third phase as the phase after the completion of the elbow extension. More particularly, we selected $25 \%$ of the duration of the reaching motion selected after the velocity drops below a threshold. The velocity threshold was set at $10 \%$ of the maximum angular velocity recorded for each subject.

For each grasp type, 10 trials were randomly selected as the testing set. The remaining 20 trials of each grasp type constituted the training set.

The preprocessed EMG signals were analyzed using a sliding time window of $150 \mathrm{~ms}$ with an increment of $50 \mathrm{~ms}$. Three features were extracted from each time window; the average (Ave), the number of slope changes (SC) and the waveform length (WFL). The features of each EMG channel were concatenated and introduced to an LDA classifier. LDA is one of the most commonly used classification algorithms for biomedical signals due to its performance and robustness. LDA finds a linearly optimal combination of the features in order to separate between classes. A fitting function estimates the parameters of a Gaussian distribution for each class and finds the probability of each point belonging to a class.

In our approach, instead of building one model for all time windows, we train three classifiers with respect to the angular velocity of the elbow joint. Specifically, we create one model for the phase with increasing angular velocity, a second model for the phase with decreasing angular velocity and a third model for the phase when the angular velocity is below the threshold. We set this threshold as the $10 \%$ of average of the peak velocity recorded from all training trials. We assume that by building a classifier for each phase, the muscular activity of the trials of the same grasp type will be more proximal to its average, which could constitute the signals more stationary, decrease the variability of the data and, thus, improve classification performance. Figure 2 presents an illustration of the approach. We compare this approach $\left(l d a_{I}\right)$ with the performance of one LDA classifier for all the phases $\left(l d a_{A}\right)$.

For further investigating the three phases, we use the squared Hellinger distance to quantify the similarity of the distributions of the classes (i.e. grasp types). The squared Hellinger distance $\left(H^{2}\right)$ between two multivariate Gaussian distributions $P \sim \mathscr{N}\left(\mu_{1}, \Sigma_{1}\right)$ and $Q \sim N\left(\mu_{2}, \Sigma_{2}\right)$ is given by the formula:

$$
H^{2}(P, Q)=1-\frac{\operatorname{det}\left(\Sigma_{1}\right)^{1 / 4} \operatorname{det}\left(\Sigma_{2}\right)^{1 / 4}}{\operatorname{det}\left(\frac{\Sigma_{1}+\Sigma_{2}}{2}\right)^{1 / 2}} e^{d}
$$

where $d=\left\{-\frac{1}{8}\left(\mu_{1}-\mu_{2}\right)^{T}\left(\frac{\Sigma_{1}+\Sigma_{2}}{2}\right)^{-1}\left(\mu_{1}-\mu_{2}\right)\right\}$

The Hellinger distance is a type of f-divergence metric, with 0 and 1 bring its lower and upper bound respectively. It reaches its maximum value (1) when the distributions do not overlap. In our case, small Hellinger distance would indicate that the mean of the distributions would be close to each other and, thus, a lager overlapping would occur between them, leading subsequently to poor classification. Whereas, large values close to 1 would indicate that the distributions are well separated from each other.

\section{RESUlTS}

\section{A. Phases of the motion and Hellinger distance}

Figure 2 a presents the Gaussian distribution of the classes on each phase for the subject 1 . The three graphs on the top the Figure 2 a correspond to the distributions of the classes when the data are projected to the space of all phases $\left(S_{A}\right)$, whereas the three graphs on the bottom correspond to the 

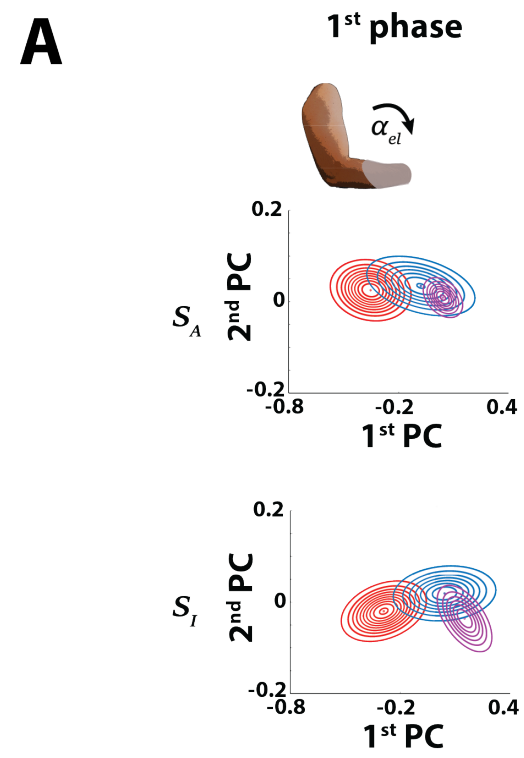

B
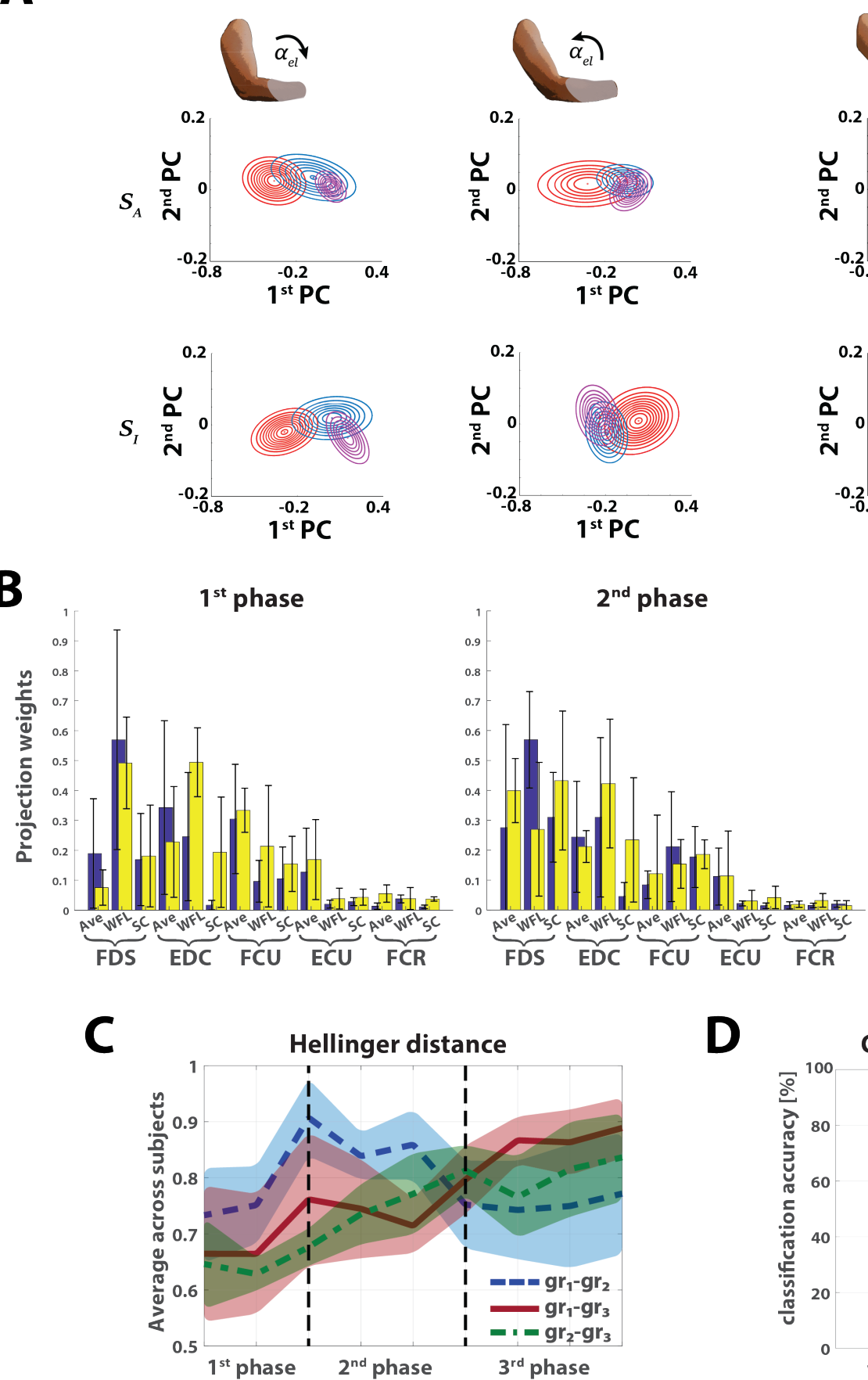
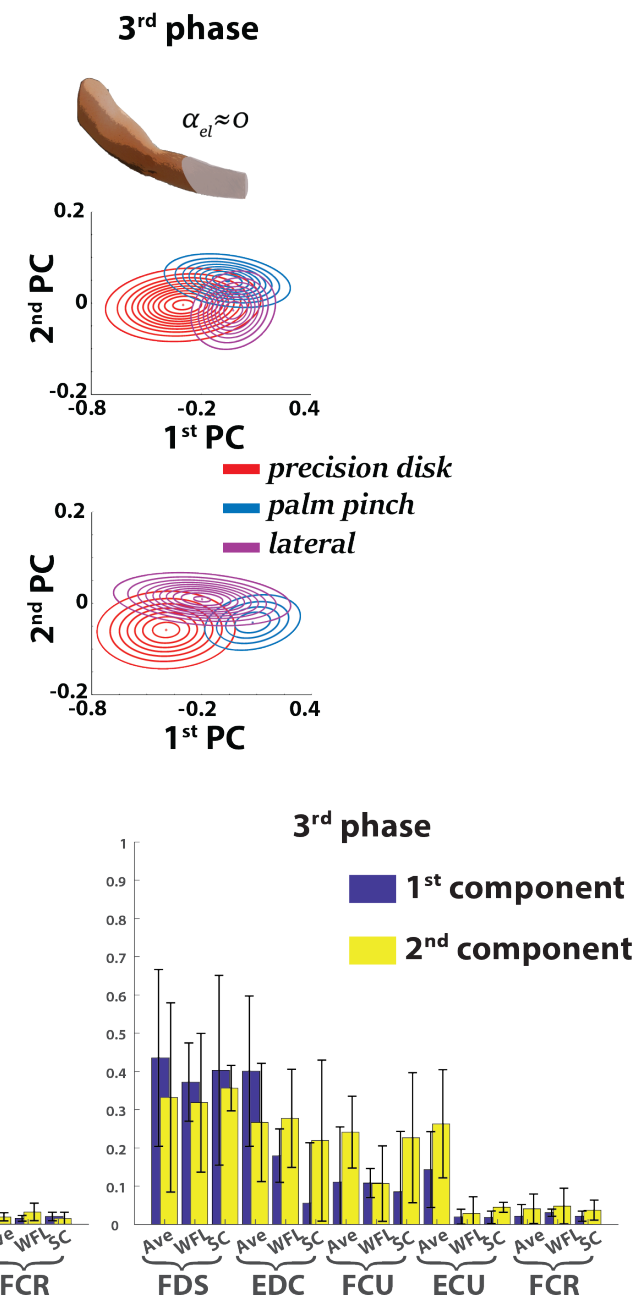

D

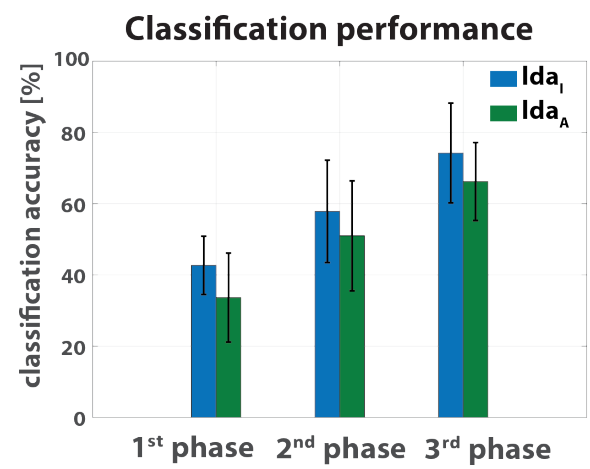

Fig. 2: a) The Gaussian distributions of the classes (i.e. grasp types) for each phase of the subject 1 on the two principal components (PC) of the LDA space. The red color corresponds to precision disk, whereas the blue and magenta color corresponds to palm pinch and lateral grasp. The three graphs on the top show the Gaussian distributions over the space of all the phases $\left(S_{A}\right)$. The three graphs on the bottom present the Gaussian distributions on the space of each phase separately $\left(S_{I}\right)$ b) The average across the subjects and the standard deviation of the projection weights after LDA and c) The average Hellinger distance across subjects between classes on all the phases. $g r_{1}, g r_{2}$ and $g r_{3}$ correspond to precision disk, palm pinch and lateral grasp respectively d)The average classification accuracy and standard deviation across subjects on the three motion phases.

distributions with the data projected on the space of each phase separately $\left(S_{I}\right)$. We observe larger overlapping among the distributions on the $S_{A}$ space than the $S_{I}$ space for all the phases. A representative example of this is the distribution of lateral grasp in the first motion phase, which is completely overlapped by the distribution of the palmar pinch on space $S_{A}$. However, the distribution of the lateral grasp is partially overlapped by the one of the palmar pinch on the space $S_{I}$.

The projection weights on the new hyperplane after LDA are presented in Figure 2b for all the motion phases. The distribution of weights is different in each phase; the wavelengths of the muscles FDS and EDC have larger values on the $1^{\text {st }}$ phase, whilst the weight of the average value of FDS increases significantly on the $2^{\text {nd }}$ and $3^{\text {rd }}$ phase. The distribution of weights is more balanced in the $3^{\text {rd }}$ phase across the muscles FDS, EDC, FCU and ECU. FCR has the smallest value among the muscles in all the phases. This could be explained by the fact that FDS and EDC 
are primarily responsible for the motion of 3 fingers; index, middle and ring fingers.

The Hellinger distance, presented on Figure 2k, indicates that the distributions of the classes are better separated in the late stages of the reaching motion. More specifically, $H^{2}$ increases in all the phases for the pairs precision disk-lateral grasp and lateral grasp-palm pinch. The $H^{2}$ between the class distributions of precision disk and lateral grasp has a large value between the $1^{\text {st }}$ and $2^{\text {nd }}$ phase, whilst decreasing in the $2^{\text {nd }}$ and increasing in the $3^{\text {rd }}$ phase.

\section{B. Classification performance}

In this part, instead of building one classifier for all the phases, we trained one classifier for each phase. Figure $2 \mathrm{~d}$ shows the average classification accuracy and standard deviation among subject for each phase. The approach with the three classifiers $\left(l d a_{I}\right)$ outperforms the one classifier in all the phases $\left(l d a_{A}\right)$. Specifically, $l d a_{I}$ has an accuracy of $42.7 \pm 8.2 \%, 57.8 \pm 14.4 \%$ and $74.2 \pm 14 \%$ in the first, second and third phase accordingly. Whereas, $l d a_{A}$ presents an accuracy of $33.6 \pm 12.5 \%, 51 \pm 15.4 \%$ and $66.2 \pm 11 \%$ for each phase accordingly.

\section{DISCUSSION}

Following our previous work [1], we explore the concept of motion phases on the EMG signals and its potentials on addressing the variability of the signals. We extend our previous analysis providing insights on the LDA projection and quantifying the similarity of the distributions of the classes (i.e grasp types) with the Hellinger distance.

Different arm movements reflect on different patterns in muscular activity in able-bodied individuals [3]. Those different EMG patterns are produced due to gravity and inertia compensation but also the fingers' motion during the hand's pre-shape. Our work is complementary to previous approaches focusing on the EMG patterns on individuals with below-the-elbow amputation, where no finger-motion occurs. Moreover, we offer insights on the EMG patterns of each class (i.e. grasp type) and quantify their evolution over time.

The arm motion introduces variation in the EMG patterns and affects dramatically the classification accuracy [3], [4], [5], [9]. Our outcomes are aligned with those findings; the arm extension in the reach-to-grasp motion creates confounds that influence the classification performance regardless of the fact that our experimental protocol involves extension of the arm towards a specific direction.

Specifically, the different projection weights (see Figure 2p) revealed that the EMG activity differs in each phase. During the reaching motion (phase 1 and 2), a dynamic contraction of the muscles occurred for compensating the gravity and inertia of the arm. This factor increased the overlapping of the class-distributions (see Figure $2 \mathrm{a}$ ) and results in small values of the Hellinger distance (see Figure $2 \mathrm{k}$ ). In the case the user wears a prosthetic device, the gravity compensation of a larger weight could have a greater impact on the EMG patterns and result in even harder separation of the classes.

As no pre-shape occurs in transradial amputees, they potentially contracted the muscles but solely to close their phantom hand. This led to the generation of more stationary EMG signals from the forearm muscles close to end of reaching motion and after it (e.g. late phase 2 and phase $3)$. As the muscle contractions became gradually isometric, the Hellinger distance (see Figure 2k) presented larger values in two of the three cases and, thus, the classes become more separable.

The changes on the EMG patterns over the motion phases had an effect on the classification performance. To examine the improvement of the classification accuracy, we compared the performance when an LDA classifier is trained for each phase with the performance of one LDA classifier in all the phases. The former approach with the three classifiers presented higher accuracy for all the phases, indicating an improved encapsulation of the EMG patterns on each motion phase.

Commercially available hand-myoprosthesis require approximately one second to fully close. Moreover, the a selfpaced reaching motion lasts $1-1.5 s$. An accurate identification of the grasping intention during the second motion phase, and thus an activation of the prosthesis on this stage, could improve the coordination with the arm motion.

A direct extension of the proposed approach would be the introduction of the kinematics of the arm, towards a multi-sensor pattern-recognition system. In the future online implementation of this work, the angular position or velocity could be included as a parameter of the system. This would provide information regarding the motion phase in real-time for selecting the proper classifier. The introduction of different hand orientations and an additional wrist control could be a further expansion of the approach.

\section{REFERENCES}

[1] I. Batzianoulis, N. E. Krausz, A. M. Simon, L. Hargrove, A. Billard, Decoding the grasping intention from electromyography during reaching motions, in Journal of NeuroEngineering and Rehabilitation, vol. 15, 2018

[2] T. Lorrain, N. Jiang, D. Farina, Influence of the training set on the accuracy of surface EMG classification in dynamic contractions for the control of multifunction prostheses, Journal of NeuroEngineering and Rehabilitation, vol. 9, 2011

[3] J. Liu, D. Zhang, X. Sheng, X. Zhu, Quantification and solutions of arm movements effect on sEMG pattern recognition, Biomedical Signal Processing and Control, vol. 13, 2014.

[4] D. Yang, Y. Gu, L. Jiang, L. Osborn, H. Liu, Dynamic training protocol improves the robustness of PR-based myoelectric control, Biomedical Signal Processing and Control, vol. 31, 2017.

[5] A. Krasoulis, I. Kyranou, M. S. Erden, K. Nazarpour, S. Vijayakumar, Improved prosthetic hand control with concurrent use of myoelectric and inertial measurements, Journal of NeuroEngineering and Rehabilitation, vol. 14, 2017.

[6] T. A. Kuiken, G. Li, B. A. Lock, R. D. Lipschutz, L. A. Miller, K. A Stubblefield, K. B. Englehart, Targeted Muscle Reinnervation for Realtime Myoelectric Control of Multifunction Artificial Arms, in JAMA, 2009.

[7] T. Feix, J. Romero, H.-B. Schmiedmayer, A. M. Dollar, D. Kragic, The GRASP Taxonomy of Human Grasp Types, in IEEE Transactions on Human-Machine Systems, 2015.

[8] Rand MK, Shimansky YP, Stelmach ABMI, Stelmach HE, Quantitative model of transport- aperture coordination during reach-to-grasp movements, Exp Brain Res. 2008.

[9] Y Gu, D. Yang, Q. Huang, W. Yang, H. Liu, Robust EMG pattern recognition in the presence of confounding factors: features, classifiers and adaptive learning, in Expert Systems with Applications, vol. 96, 2018 , 